\title{
Bauimmissionen und Klinikbetrieb - ein praktischer Lösungsansatz
}

\author{
Walter Aus der $A u^{a}$, \\ Rolf P. Steinegger ${ }^{b}$ \\ a Dr. med., Facharzt für Ophthal- \\ mologie FMH, insbesondere \\ Ophthalmochirurgie, Bern \\ b Fürsprecher, Bern
}

Korrespondenz: Rolf P. Steinegger Steinegger Rechtsanwälte Hirschengraben 2 Postfach 8364 CH-3001 Bern Tel. 0313820301 Fax 0313820482

info@sb-law.ch

www.sb-law.ch

\begin{abstract}
Ausgangslage
Der vorliegende Aufsatz stellt ein Modell dar, wie eine Klinik, die im Mikrochirurgiebereich arbeitet, trotz laufender Bauarbeiten im gleichen Haus weiterbetrieben werden kann.

Eine Grundeigentümerin in Bern beabsichtigt, ihre Liegenschaft, ein Mehrfamilienwohnhaus mit zahlreichen 1-Zimmer-Studios, umzubauen und zu sanieren. Im gleichen Baukomplex befindet sich eine Augenklinik für Refraktiv- und Kataraktchirurgie. Das Bauvorhaben wird mit Lärm und Erschütterungen verbunden sein, die den Betrieb der Augenklinik während der Bauzeit von ein bis zwei Jahren in Frage stellen.
\end{abstract}

\section{Baueinsprache}

Die Augenklinik erhebt gegen das Baugesuch Baueinsprache mit Rechtsverwahrung und verlangt Massnahmen [1, 2] in bezug auf:

- die Bauarbeiten;

- die lärmintensiven Bauarbeiten;

- die Bautransporte und

- die Erschütterungen.

Als konstruktives Massnahmekonzept schlagen die Einsprecher vor: Vor Beginn der Bauarbeiten muss die Baugesuchstellerin durch Erschütterungs- und Lärmmessungen den Nachweis erbringen, dass die vorgesehenen Baumethoden und Arbeitstechniken den Anforderungen der Augenklinik genügen. Die Erschütterungs- und Lärmmessungen müssen durch einen neutralen Gutachter ausgeführt und dokumentiert werden.

Sollten die Resultate ungenügend sein, kann durch die folgende, im Inselspital Bern erprobte Massnahme (Umbauten in unmittelbarer Nähe des Operationstraktes, unter anderem Mikrochirurgie; ab 1998) ein sofortiger Arbeitsunterbruch angeordnet werden, wenn dies für eine Augenoperation erforderlich ist.

Anbringen von Warnblinklampen, kombiniert mit Sirenen, die nur durch die Augenklinik ein- und ausgeschaltet werden können. Die Sirenen sollen nur auf die Warnblinklampen aufmerksam machen. Die Warnblinklampen blinken nur so lange, wie auf der Baustelle keine

\section{Nuisances de chantier et exploitation d'une clinique: solutions pratiques}

L'article ci-dessous met en évidence un exemple, celui d'une clinique qui gère un domaine de microchirurgie, qui peut continuer son exploitation malgré les travaux de rénovation qui se font dans la même maison.

Une propriétaire foncière à Berne a l'intention de transformer et de rénover son bien immobilier, une maison à plusieurs étages en de nombreux studios d'une chambre. Dans le même immeuble se trouve une clinique d'ophtalmologue spécialisé dans la chirurgie réfractive et de la cataracte. La rénovation est liée à des nuisances telles que le bruit et les vibrations, pendant les travaux, remettent en question le fonctionnement de la clinique pour un à deux ans.

Dans un premier temps, la clinique ophtalmologique a formé recours contre la demande de construire, puis retiré celui-ci après quelques négociations de conciliation. Les conventions alors passées se sont avérées efficaces. Conclusion: afin de pouvoir surmonter des situations complexes et de parvenir à un bon résultat, la communication entre les parties par écrit (contrats), comme aussi par oral, est une condition sine qua non.

Arbeiten ausgeführt werden dürfen, die Erschütterungen oder Lärm verursachen. Für die Einhaltung dieser Anordnung ist die Bauleitung verantwortlich.

Als weitere Massnahmen können Sperrzeiten vereinbart werden und Arbeiten, die Erschütterungen und Lärm verursachen, in Früh- und Spätschichten ausgeführt werden. 


\section{Vereinbarung der Parteien}

Im Interesse, eine rasche Regelung zu finden, die den Gleichlauf von Klinikbetrieb und Bauarbeiten gewährleistet und die während der Bauzeit ohne Verzug durchgesetzt werden kann, nehmen die Parteien Vergleichsverhandlungen auf. Dabei lassen sie sich von Experten, namentlich von Baustatikern und Spezialisten für Bauakkustik, begleiten.

Es kann folgendes vereinbart werden:

- Allgemein: Begrenzung der Immissionen während der Bauzeit, so dass der normale Betrieb der Augenklinik gewährleistet werden kann.

- Die Bauarbeiten sollen durch einen Generalunternehmer ausgeführt werden, der über die besondere Situation im Bereich Lärm und Erschütterungen orientiert ist.

- Definition von Grenzrichtwerten für Lärm und Erschütterungen aufgrund der bestehenden Vorbelastung aus dem Betrieb der Einsprecher, von Erfahrungswerten sowie der subjektiven Beurteilung während der Testversuche unter teilweiser Mitwirkung des Einsprechers und aufgrund der sinngemäss anwendbaren SIA-Norm 181, Schallschutz im Hochbau, da keine expliziten Grenzwerte bestehen.
- Regelung des Vorgehens und der gegenseitigen Rechte und Pflichten beider Parteien während der Bauphase.

- Ausführungsbestimmungen für die Bauarbeiten.

- Begehung der Liegenschaft, anlässlich deren der Betrieb der Einsprecherin und deren Bedürfnisse vorgestellt werden.

- Die Termine der mit Lärm und Erschütterungen verbundenen Arbeiten werden wöchentlich zwischen Einsprecherin und Baustellenleitung abgesprochen.

- Die Baugesuchstellerin verpflichtet sich, eine messtechnische Bauüberwachung des Lärms und der Erschütterungen sicherzustellen, und übernimmt deren Kosten.

- Einrichtung eines Alarmmechanismus bei Übertreten der definierten Grenzwerte.

- Bestimmen einer Arbeitsgruppe «Überwachung und Alarm».

- Justierung von Lasergeräten und anderen empfindlichen Geräten. Die Kosten der baubedingten Justierungen gehen zu Lasten der Baugesuchstellerin.

- Regelung der Baustellentransporte, des Schutzes vor Staubentwicklung und des Zugangs zu der Klinik und den Parkplätzen.

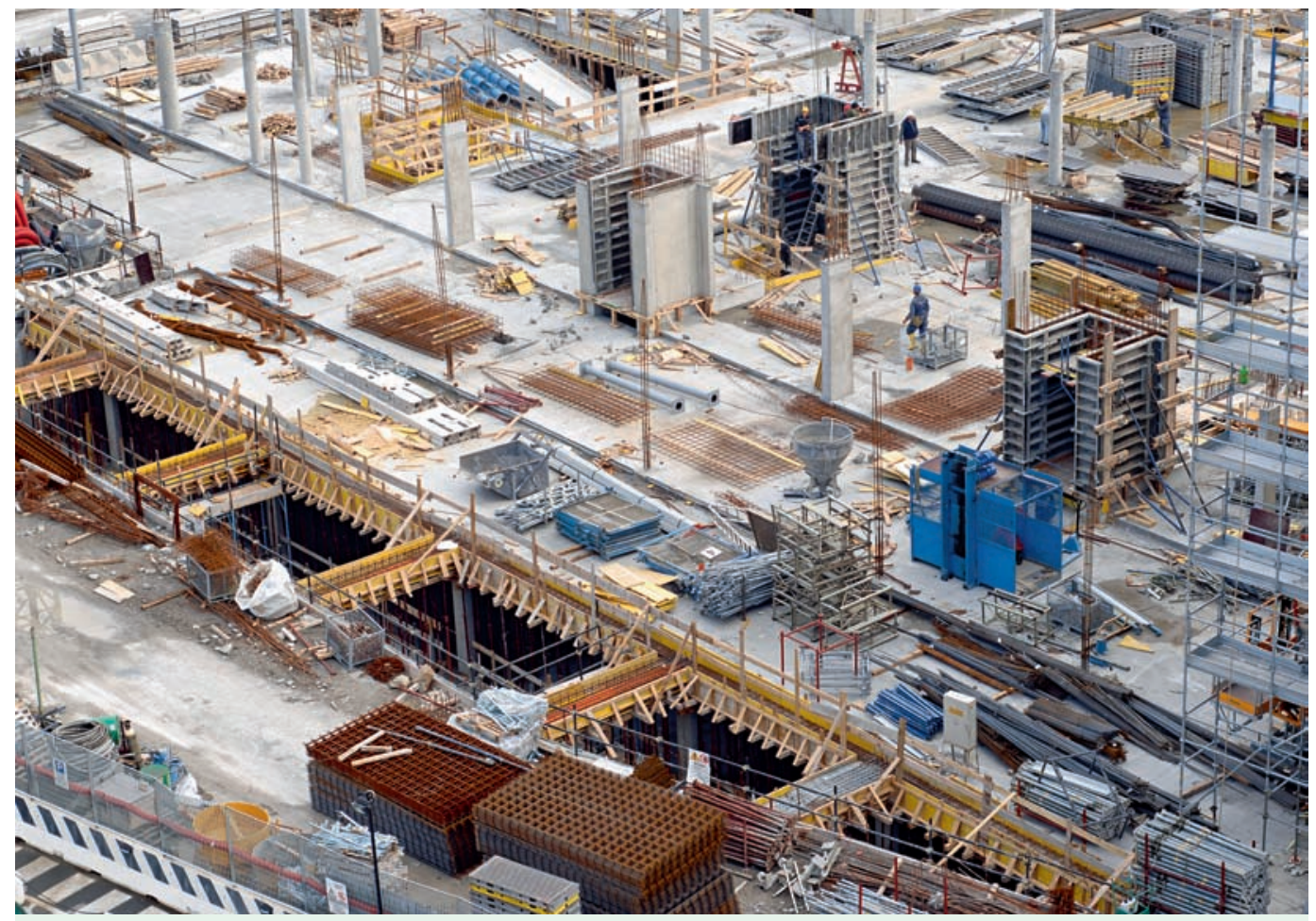

Bauarbeiten können einen Klinikbetrieb erheblich beeinträchtigen (das hier gezeigte Bild gibt nicht das im Artikel geschilderte Bauvorhaben wieder). 
- Die Zuluftfassung der Lüftung der Augenklinik wird durch einen Grobstaubfilter zusätzlich vor Verunreinigungen geschützt.

- Festlegen einer Konventionalstrafe sowohl für Missachtung der «Zeitfenster» durch die Baugesuchstellerin wie für Fehlalarme der Augenklinik.

- Entschädigung an Einsprecherin und Versicherung.

\section{Praktische Erfahrungen des Mediziners und Klinikleiters}

Die Einsprache wurde zurückgezogen; die Rechtsverwahrung blieb aufrechterhalten. Die getroffenen Vereinbarungen für das Bauprojekt erwiesen sich als äusserst effizient. Der Klinikbetrieb konnte ohne wesentliche Störungen praktisch während der ganzen Umbauphase weitergeführt werden.

Die internen Lärmmessungen erwiesen sich bald als überflüssig, war doch der interne Lärmpegel oft viel grösser als die Bauimmissionen. Trotz aller Vereinbarungen kam es aber gelegentlich zu Zwischenfällen (unerwartet hoher Lärmpegel oder Erschütterungen) von nicht orientierten Handwerkern, zweimal zu Wassereinbrüchen kleinerer Ausmasse. Diese Ereignisse erforderten jeweils eine rasche Kommunikation mit der Bauleitung und liessen sich meistens innert kürzester Zeit beheben.

Soweit möglich wurden der Bauleitung zusätzliche Zeitfenster für störende Arbeiten zuge- teilt, unter anderem während der Betriebsferien der Klinik oder anderer Arbeitsunterbrüche. Durch diese gegenseitige Kooperation war es möglich, das Bauvorhaben innerhalb der gesetzten Frist zu realisieren. Dies trotz unerwarteter Schwierigkeiten bezüglich der Bautechnik.

Das nun erzielte Resultat des Umbaues wertet die gesamte Liegenschaft inklusive der Augenklinik auf und bietet nun höchstmoderne, erstklassige Wohnungen nahe dem Stadtzentrum an.

Einmal mehr zeigte es sich mit aller Deutlichkeit, dass, um komplexere Vorgänge zu beherrschen und dadurch zu einem guten Resultat zu kommen, Kommunikation unter den Parteien in schriftlicher (Verträge), genauso aber auch in mündlicher Form eine Conditio sine qua non darstellt.

Hinweis: Interessierte erhalten den vollständigen Vergleichstext über den Zweitautor oder den FMH-Rechtsdienst (lex@fmh.ch).

\section{Literatur}

1 Unter Verweis auf den Aufsatz von Peter Hänni/ Jörg Schmid, Bauimmissionen - ein Problem des öffentlichen und privaten Rechts, in Baurechtstagung, Freiburg 1997, Band 1, S. 52ff., und die Baulärmrichtlinie des BUWAL vom 2.2.2000.

2 Die Nichteinhaltung öffentlich-rechtlicher Immissionsvorschriften, die für die Bauphase gelten, ist auch für die Beurteilung der Übermässigkeit nach Art. 684 ZGB relevant (Baurechtstagung Freiburg, 1997, a.a.O., 66). 